\title{
Protein profiles derived by automated immune precipitation
}

\author{
ROBERT F. RITCHIE
}

From the Rheumatic Disease Laboratory (Department of Research), Maine Medical Centre, Portland, Maine, United States of America

This presentation will cover much the same ground as Professor Laurell's but from a slightly different viewpoint. There is no great difference between us, though we use agarose gel electrophoresis only to detect paraproteins. Like others, we feel that scanning the electrophoretogram contributes nothing to the interpretation of the test ${ }^{1}$, as even with high resolution systems the various fractions are composed of mixtures. Instead, we prefer to estimate specific proteins by the automated immunoprecipitation system, and we routinely estimate 12 plasma proteins (albumin, $\alpha_{1}$-antitrypsin, haptoglobin, orosomucoid, $\alpha_{2}$-macroglobulin, transferrin, complement C3, complement C4, IgG, IgA, IgM, LDL protein) and can estimate more than 30 . The same proteins could of course be estimated by the techniques outlined by Dr Laurell. Specific protein analysis is superior to electrophoresis in that in addition to giving information on individual proteins it is quantitative. It is also reliable over a wide range of concentrations, it will differentiate proteins which occupy the same electrophoretic zone, and it is applicable to samples which may be unsatisfactory for electrophoresis due to haemolysis, etc. It cannot, however, replace electrophoresis in the identification of small paraproteins, particularly of free light chains which cannot be detected with the present AIP system, nor in the study of genetic polymorphism when the total amount of protein in different specimens may be the same even though they contain different molecular variants.

Before discussing the interpretation of the profiles obtained by specific estimation of 12 proteins using current methods, it is pertinent to consider the quality of the analytical results obtained. About 18 months ago, the Centre for Disease Control in Atlanta, Georgia, distributed to many laboratories in the United States three samples of serum containing known amounts of $\operatorname{IgG}, \operatorname{IgA}$, and $\operatorname{IgM}$ for analysis. The great majority of the laboratories used

${ }^{1}$ The importance of scanning for the estimation of paraproteins is emphasized by Dr Carter and Dr Kohn. radial immunodiffusion, and a few used the AIP system. The results for IgG (fig 1) were poor, to say the least; whereas the actual value was about 11.5 $\mathrm{g} / \mathrm{l}$, the observed values ranged from $7 \cdot 5$ to $18 \mathrm{~g} / \mathrm{l}$. Results from the five reference laboratories were somewhat better than this. The results for $\operatorname{IgA}$ were similar; for one sample containing about $1.45 \mathrm{~g} / \mathrm{l}$ IgM, some laboratories found less than $0.75 \mathrm{~g} / \mathrm{l}$ and others more than $3.0 \mathrm{~g} / \mathrm{l}$, although the reference laboratories were all close to the true figure. For another specimen with an IgM content of about $45 \mathrm{~g} / 1$ a large number of laboratories found the IgM content to be zero and only a few laboratories obtained values within an acceptable range (fig 1). Had the operator merely looked at the sample, it would have been obvious that there was something seriously abnormal because the sample had the consistency of syrup. Electrophoresis would have shown that almost all of the plasma protein was IgM.

There are probably several causes of these poor results, including faulty technique, but in this particular circumstance the companies which manufacture the plates containing antibody must take a share of the blame.

Interpretation of the results of protein estimation is almost always based on a comparison of values expressed in $\mathrm{g} / 1$ or units, with a 'normal range' determined by measurements on a heterogeneous population. However, a number of factors will influence the levels found in such a population and must be taken into account when results are interpreted. Most of us would accept that age, ethnic group and sex all influence the serum levels of the immunoglobulins. Other factors include upper respiratory and gastrointestinal viral infection, pregnancy and oral contraceptives.

When considering individual proteins the question arises whether to correct for the total protein concentration which may be falsely lowered, eg, by parenteral administration of fluids, or falsely elevated, eg, by venous stasis during sampling or evaporation in the laboratory. The problem remains whether the observed value for total protein 


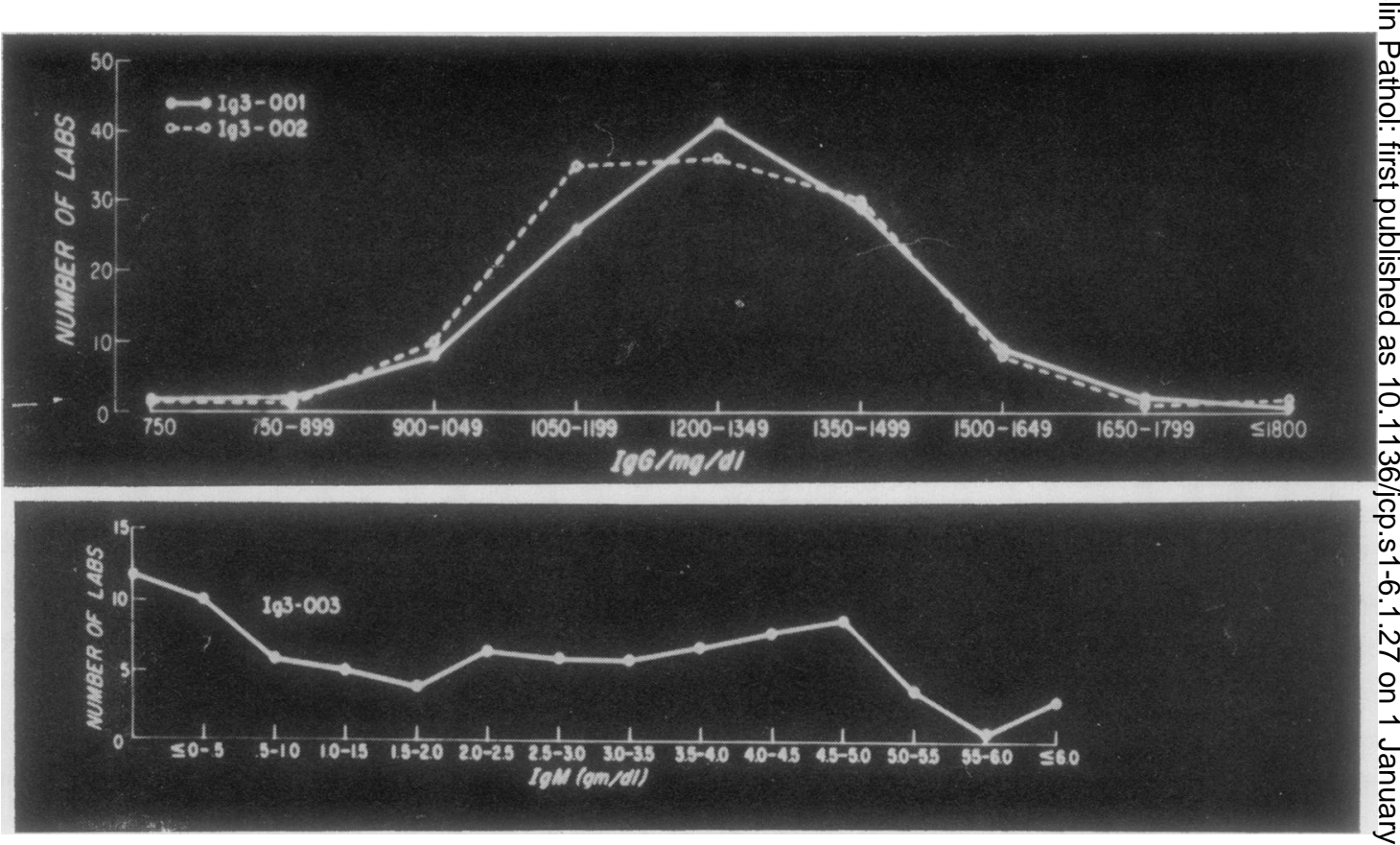

Fig 1 Results obtained for IgG and IgM in sera distributed to many laboratories. Ig3-001, Ig3-002, and Ig3-00ख्य represent three different sera.

should be used or the value obtained after subtracting the amount of any paraprotein from the total.

Ideally, interpretation should be based on the normal values for the particular individual, but such information is rarely available, in contrast to clinical data such as the patient's normal height and weight, etc. Well-person laboratory screening will necessary to provide such base-line values.

The limitations of a normal range determined in population is illustrated by the results of serum protein estimation in 2200 randomly selected people (fig 2). The distribution is very wide for some pro를

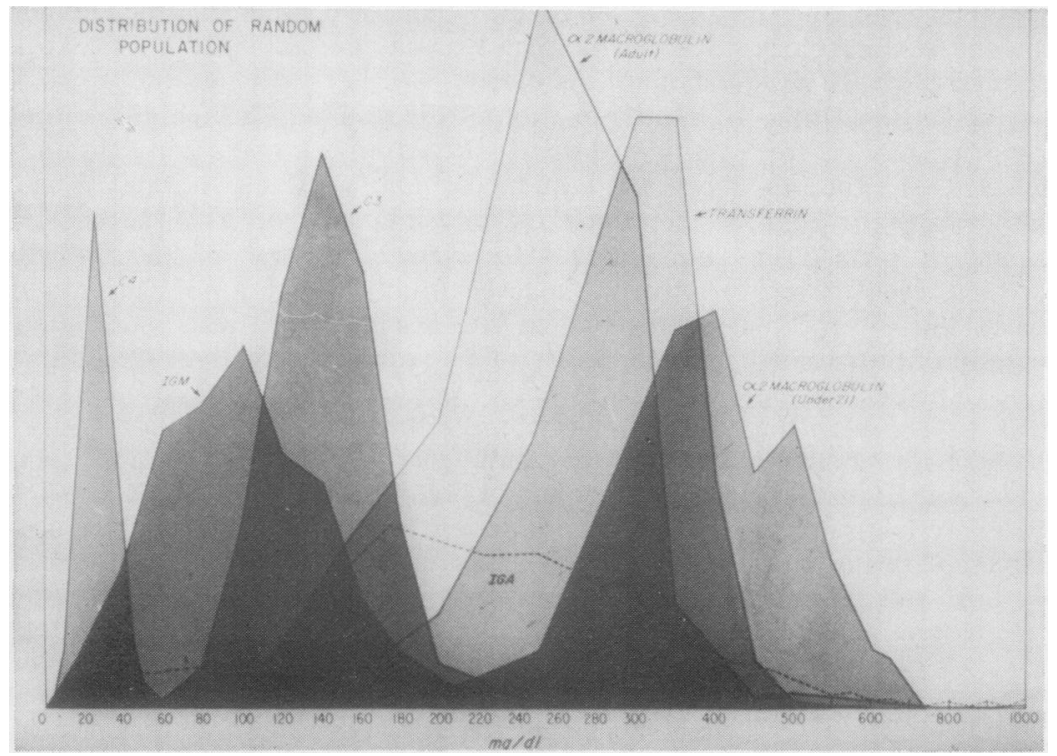

Fig 2 Distribution of serum protein concentrations in 2200 randomly selected people. 
teins, eg, IgA. An even wider range could result if measurements happened to be made during a period when there were many 'normals' with subclinical upper respiratory or gastrointestinal infections. Some proteins, such as $\alpha_{2}$-macroglobulin, have a bimodal distribution corresponding with subjects over or under 21 years of age, but the ranges in the two age groups overlap. Thus, a population norm is only of limited value for interpreting data from an individual, but at the moment it is all that is available.

A further difficulty in determining the normal range is that of deciding whether all values obtained

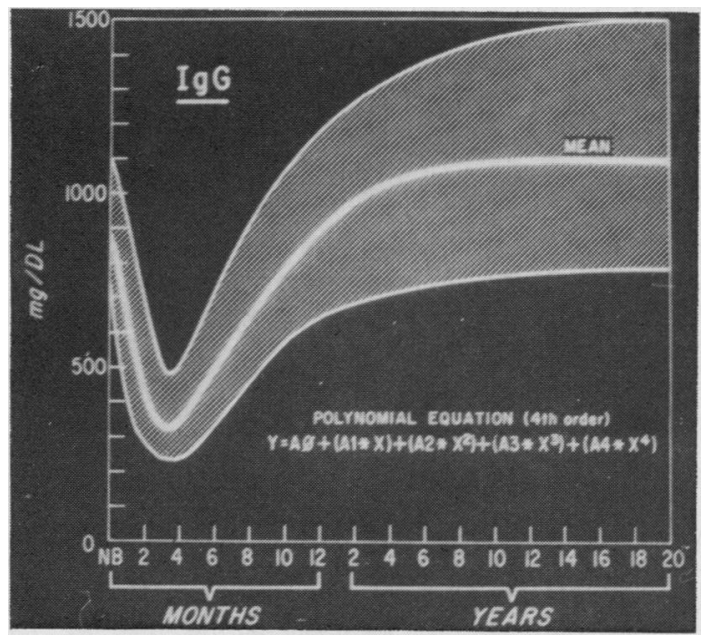

FIG. 3 from an apparently normal population should be included, whether all values from an apparently abnormal population should be excluded, and whether the criterion of 'normality' should be extended to the relatives of the individuals studied. For example, the range of values obtained for serum low-density lipoproteins is very wide, but when values from subjects who have relatives under 55 years of age with cardiovascular disease are excluded, the range is much narrower, although there are still some outliers. Our data on low-density lipoproteins show a distinct difference between the prepubertal individual, the middle-aged and the aged. We have a fairly large population aged over 80 in northern New England and we found that the low-density lipoprotein levels for those aged over 70 approximated to those of adolescents, the levels in middle age being somewhat higher. This raised the possibility that those with the higher values in middle age had less chance of reaching the 70 s, and posed the question, Should they be treated prophylactically? We found outliers, even in children, supporting the notion that the latter may have biochemical disease for two or three decades before the first signs of coronary or cardiovascular disease become manifest; if so, low-density lipoprotein estimations in childhood may enable protective measures to be taken.

It is well known that the concentrations of many proteins alter dramatically in early life, so that in order to interpret any given value properly it is necessary to know the age of the individual. The changes in IgG and IgM are shown in figures 3 and

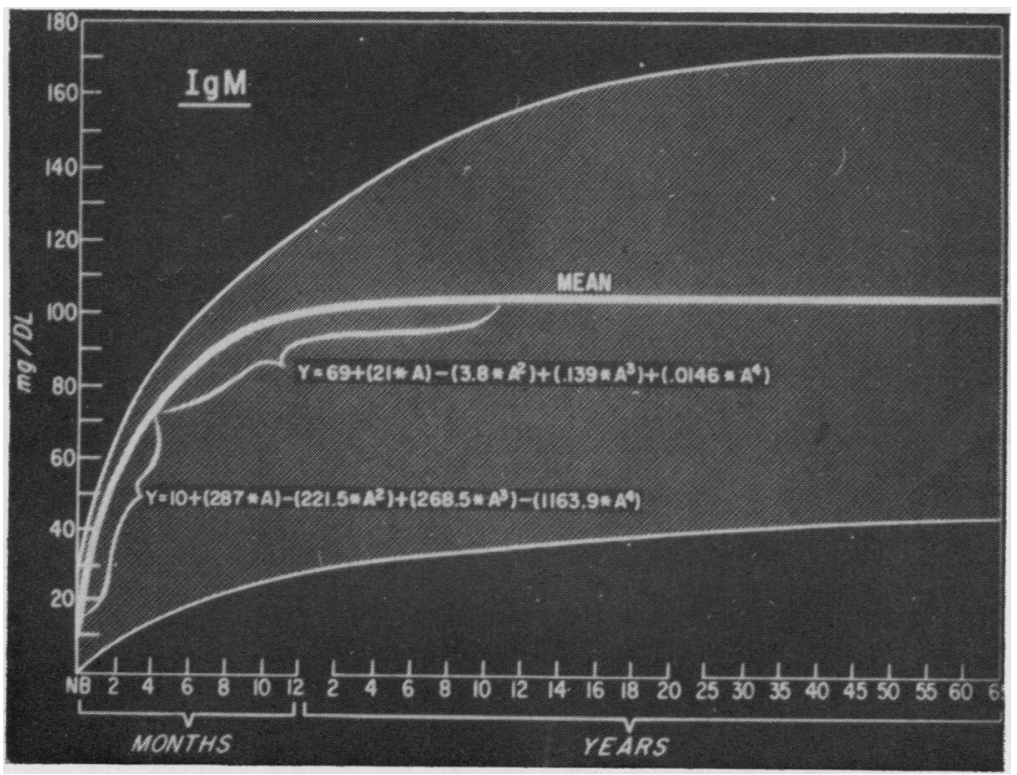

Fig 3 Variation of serum IgG concentration (ordinate) with age (abscissa).

Fig 4 Variation of serum IgM (ordinate) concentration with age (abscissa). 
4. In addition to large changes with age, which make interpretation of IgG levels particularly difficult, there is also a very wide range of values at any given age. In order to evaluate the benefit that would be gained by following an individual's values from year to year, we studied individuals at three monthly intervals for many years. The variation was very small in the absence of intercurrent illness; for example, for IgG we found variations of 1.0 to $1.5 \mathrm{~g} / 1$ as compared with the overall range of $8.0 \mathrm{~g} / 1$ for a large population. The fact that the physiological variation in an individual is so much less than the range of values found in a population seriously compromises our ability to interpret a single set of values.

We have attempted to assist the physician to overcome some of the difficulties of interpreting laboratory data by displaying them in a manner which is easy to read, and which does not require knowledge of the numerical values or of the normal range. This takes the form of a computer printout, with a bar representing the protein level as a percentage of the normal mean (for the population) running across a colour-shaded background (fig 5); the central zone denotes approximately the normal mean \pm 1 standard deviation, the intermediate zones \pm 1 to 2 standard deviations, and the outer zones any deviation exceeding \pm 2 standard deviations. The mean and standard deviations are adjusted by the computer for various factors where appropriate, such as age and sex. In the case of haptoglobin the distribution of values in a large population is very skewed, so that the normal range cannot be indicated by means of the standard deviation. Our population mean for haptoglobin is about $1.05 \mathrm{~g} / 1$, and $0.2 \mathrm{~g} / 1$ is still within the normal range. However, if the latter value were displayed in terms of the mean and standard deviation, it would $\%$ appear to be an abnormally low result. Therefore, in $\overrightarrow{\vec{F}}$ order to display the result in the standard manner, $\overrightarrow{0}$ we have to adjust the value for the skewed distribu- $\frac{}{0}$ tion by means of an equation which brings the result $\frac{\bar{\sigma}}{\bar{c}}$ into the low-normal range (fig 5).

Interpretation of the results by computer is an elaborate process (fig 6). The interpretative pro- $\infty$ gramme involving only 10 of the 12 proteins amounts $\vec{O}$ to about 150000 words and requires about $15 \overrightarrow{\vec{H}}$ seconds to process. The computer may print out upw to two pages of information, which is of course too much for anyone to read. The machine goes through? about 800 individual decisions, some of which are illustrated in figure 7 . In the example shown the? first question is, "Is the IgG value less than or $\vec{\sim}$ greater than $40 \%$ of the "normal", taking account of age etc?' If it is less than $40 \%$ the computer would print out 'marked hypoimmunoglobulinaemia in- $\vec{c}$ volving IgG'. If it is greater than $40 \%$ but less than $65 \%$ a statement 'significant hypoimmunoglobulinaemia involving IgG' would be printed. These values for 40 and $65 \%$ are, of course, only relevant $\vec{\omega}$ to our particular population.

The importance of correcting for total proteing illustrated by the profile in figure 7 . At first sight looks abnormal and was reported as such. Howeve्e, we would now correct this for the raised total pro-o tein, which would reduce all the values into the $\frac{0}{\infty}$ normal zone. The new printout would now say that $\propto$ this study is normal, but would also state that there $\overrightarrow{\overrightarrow{\vec{A}}}$ is probably haemoconcentration. When to correct 3 for alterations in total protein concentration is very

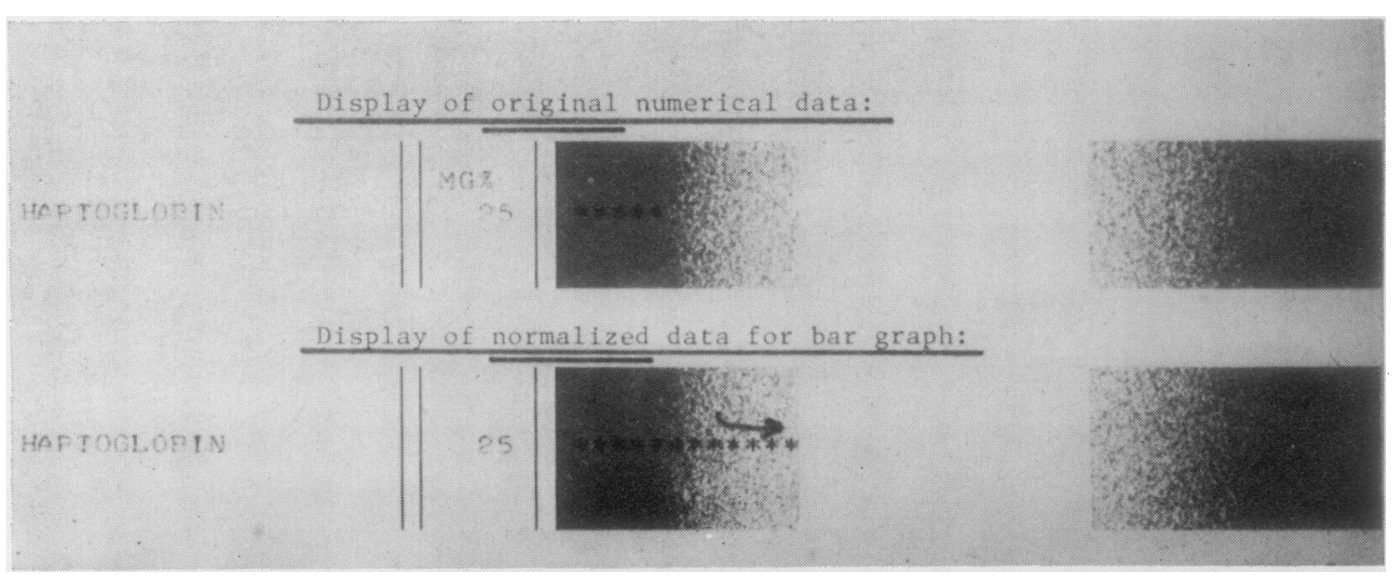

Fig 5 Illustration of the computer printout of serum protein values. The central zone indicates the mean $\pm 1 S D$ for a normal population, the intermediate grey zone $\pm 1 S D$ to $\pm 2 S D$. The lower display illustrates the effect of a correction by the computer for the skewed distribution of the normal range for haptoglobin. 


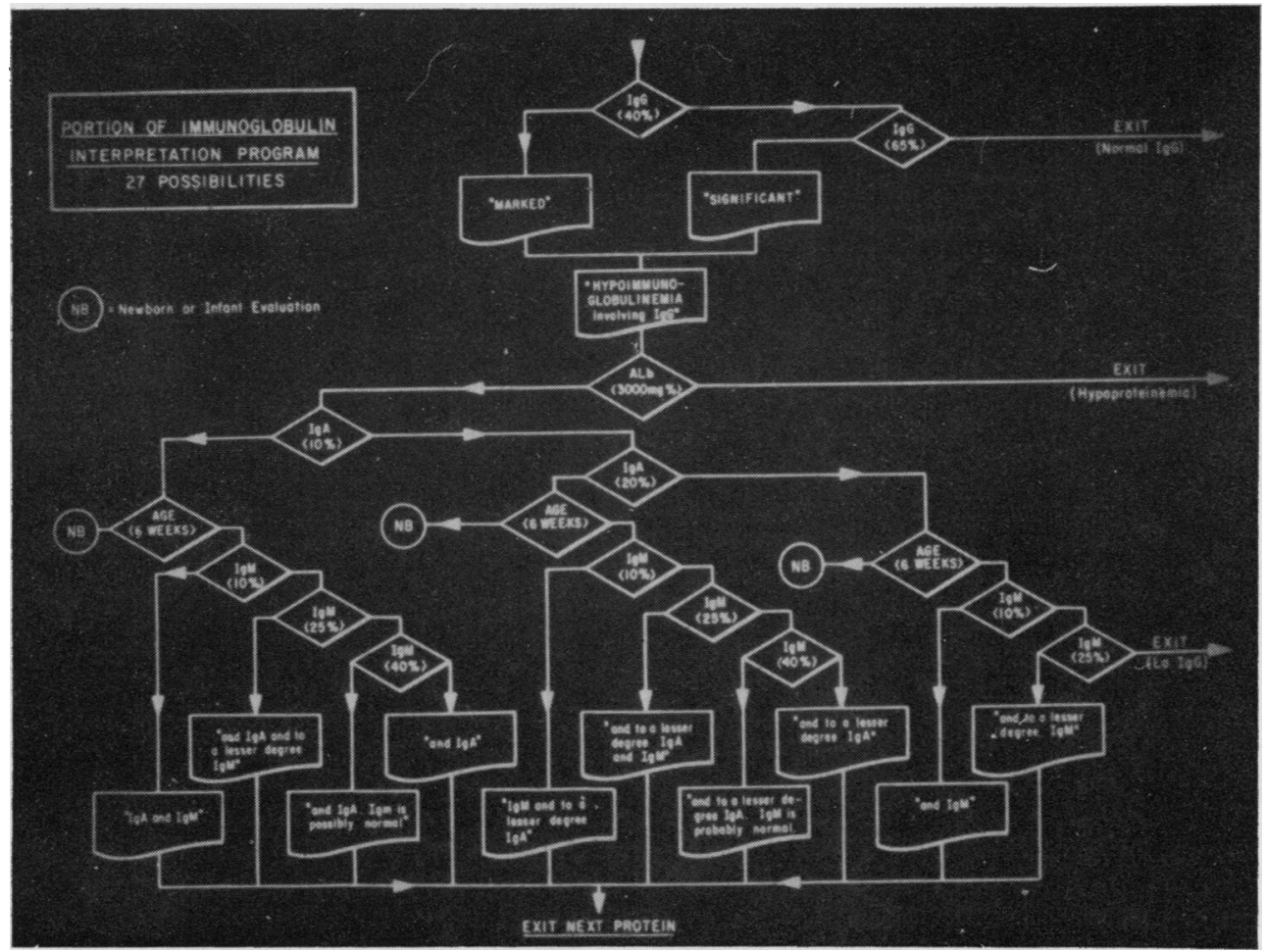

Fig 6 Part of the interpretative program for the computer.

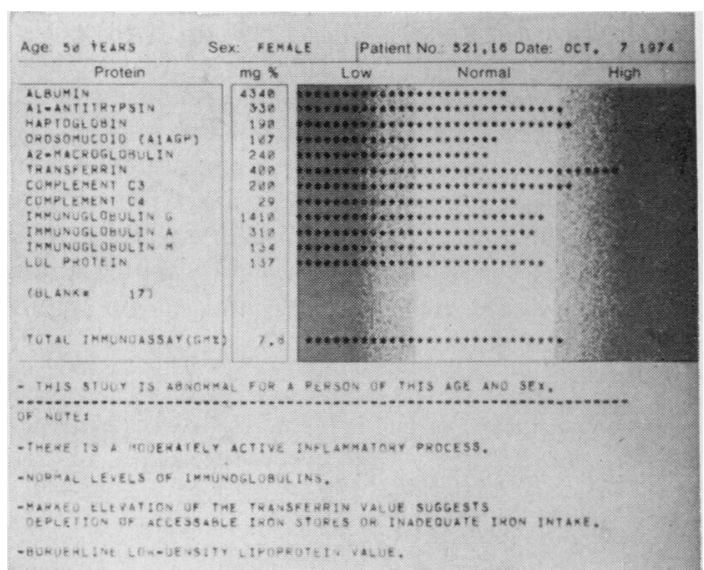

Fig 7 Example of computer printout ${ }^{1}$.

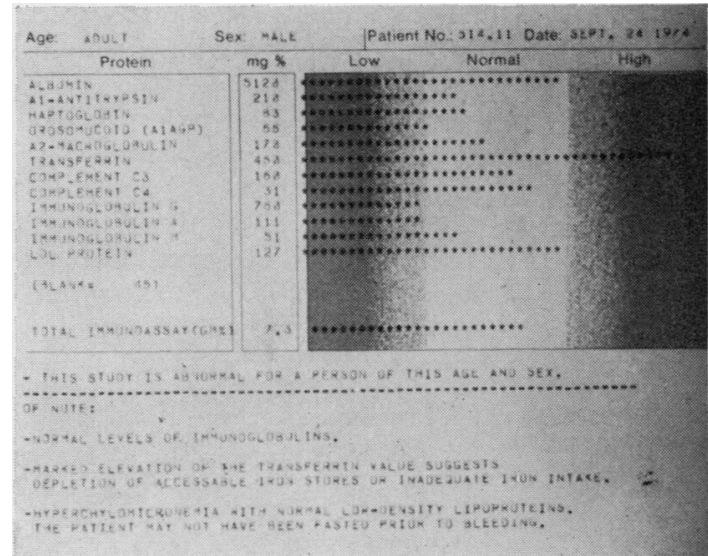

Fig 8 Example of computer printout ${ }^{1}$.

${ }^{1}$ The author has been unable to provide better photographs of the printouts illustrated in figs $7-10$ but readers should be able to see the pattern in each. 


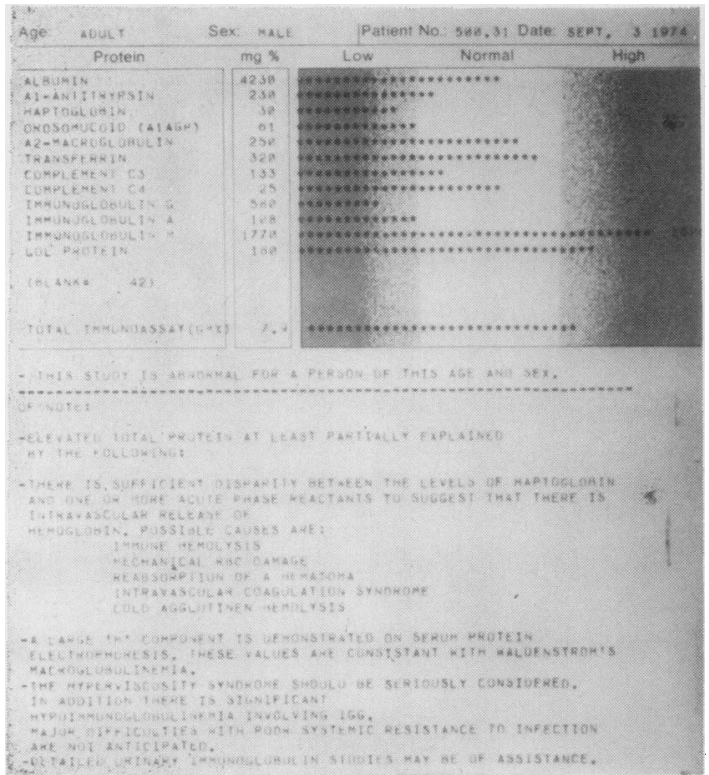

Fig 9 Example of computer printout ${ }^{1}$.

difficult to assess and to program for but seems to be very valuable.

The situation illustrated in fig 8 is somewhat different. Elevation of transferrin is the only abnormality, and this is an unusual finding in a male. The commonest two conditions in which we have seen this are chronic blood loss and lymphoma.The presence of hyperchylomicronaemia is also noted, probably indicating that the patient was not fasting; this was deduced from the blank value in the system which gives a fairly good estimate of serum triglyceride levels.

Sometimes the report has to be more detailed. An example is given in fig 9 which shows $\alpha_{1}$ antitrypsin falling in the normal zone, haptoglobin falling somewhere below it, and orosomucoid virtually normal. The computer has stated that there is a disparity between the level of haptoglobin and one or more of the other acute phase reactants.

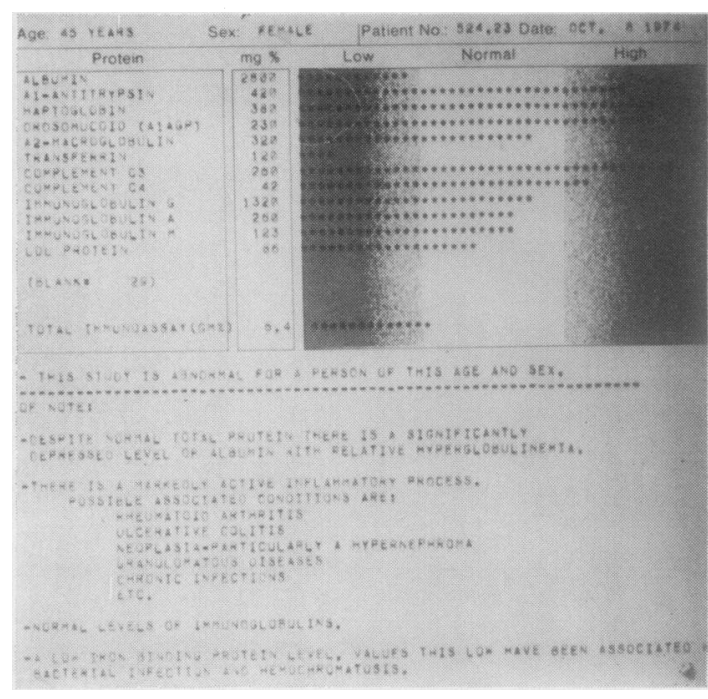

Fig 10 Example of computer printout ${ }^{1}$.

In a case of very low transferrin accompanied by $\vec{\oplus}$ only slight reduction in albumin (fig 10) the computer. concluded that the low transferrin could be associated with bacterial infection, and suggested othe possibilities. If the albumin were markedly reduced, a protein-losing syndrome would be more likely.

In addition to analysing such qualitative changes $\frac{\mathbb{}}{\Phi}$ in the protein pattern, the computer can also be $\vec{F}$ programmed to take account of the diagnostic 3 significance of the degree of change in the levels of individual proteins.

If progress in this field is to continue, it is undoubtedly necessary to stimulate wider interest and? to accumulate more data which must be technically reliable. This requires proper training of technicians, suitable instruments and reliable reagents. The clinician must be appraised of the clinical indications for making these measurements and of the significance of the results. 